$$
\text { CONF- } 9605160 \cdots 1
$$

\title{
A Simple Model for Cyclic Variations in a Spark-Ignition Engine
}

\author{
C. S. Daw, J. B. Green, Jr, M. B. Kennel, J. F. Thomas \\ Oak Ridge National Laboratory \\ Oak Ridge, TN 37831
}

\author{
C. E. A. Finney \\ University of Tennessee \\ Knoxville, Tennessee 37996-2210 \\ F.T. Connolly \\ Ford Motor Company \\ Deartorn, Michigan 48121-2053
}

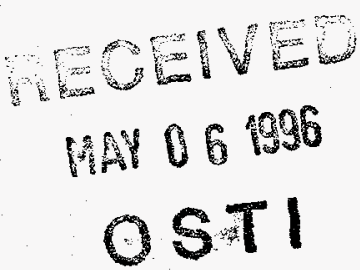

To Be Presented

To

The Central States Section

1996 Technical Meeting

of

The Combustion Institute

May 5-7, 1996

St. Louis, Missouri

"The submitted manuscript has been authored by a contractor of the U.S. Government under contract DE-ACOS-960R22464. Accordingly, the U.S. Government retains a nonexclusive, royalty-free license to publish or reproduce the published form of this contribution, or allow others to do so, for U.S. Government purposes."

\section{DISCLAIMER}

\author{
Prepared by the \\ Oak Ridge National Laboratory \\ Oak Ridge, Tennessee 38831-8088 \\ Managed by \\ Lockheed Martin Energy Research Corp. \\ for the \\ U.S. Department of Energy \\ under contract DE-AC05-960R22464
}

This report was prepared as an account of work sponsored by an agency of the United States Government. Neither the United States Government nor any agency thereof, nor any of their employees, makes any warranty, express or implied, or assumes any legal liability or responsibility for the accuracy, completeness, or usefulness of any information, apparatus, product, or process disclosed, or represents that its use would not infringe privately owned rights. Reference herein to any specific commercial product, process, or service by trade name, trademark, manufacturer, or otherwise does not necessarily constitute or imply its endorsement, recommendation, or favoring by the United States Government or any agency thereof. The views and opinions of authors expressed herein do not necessarily state or reflect those of the 


\section{DISCLAIMER}

Portions of this document may be illegible in electronic image products. Images are produced from the best available original document. 


\title{
A SIMPLE MODEL FOR CYCLIC VARIATIONS IN A SPARK-IGNITION ENGINE
}

\author{
C.S. Daw*, J.B. Green, Jr., J.F. Thomas, M.B. Kennel, \\ Oak Ridge National Laboratory, Oak Ridge, TN 37831-8088 \\ C.E.A. Finney, University of Tennessee, Knoxville, Tennessee 37996-2210 \\ F.T. Connolly, Ford Motor Company, Dearborn, Michigan 48121-2053
}

\begin{abstract} agreement with our experimental data.

\section{Introduction}

Ever since the investigations of Clerk [1], researchers have reported apparently conflicting observations of cyclic combustion variations in spark-ignition engines. With increasing emphasis on lean fueling and exhaust gas recirculation to minimize $\mathrm{NO}_{x}$ emissions, cyclic variability (also known as cyclic dispersion) has received renewed attention. Examples of this recent interest can be found in [2-12]. In most studies, cyclic variability has been described as either stochastic (random) or deterministic in nature. We propose a model that combines both stochastic and deterministic elements. We avoid complex spatial details, opting instead to focus on the global combustion process and how that process evolves under the combined influence of stochasticity and determinism over thousands of engine cycles.
\end{abstract}

We propose a simple model that explains important characteristics of cyclic combustion variations in spark-ignited engines. A key model feature is the interaction between stochastic, small-scale fluctuations in engine parameters and nonlinear deterministic coupling between successive engine cycles. Prior-cycle effects are produced by residual cylinder gas which alters mean in-cylinder equivalence ratio and subsequent combustion efficiency. The model's simplicity allows rapid simulation of thousands of engine cycles, permitting in-depth statistical studies. Additional mechanisms for stochastic and prior-cycle effects can be added to evaluate their impact on overall engine performance. We find good

\section{The Model}

Our basic assumptions are as follows. - We consider a single cylinder with injection of fresh air and gaseous (vaporized) fuel prior to combustion.

- Total gas moles in the cylinder prior to combustion are constant.

- Residual gas (post-combustion gas from previous cycle) mixes with fresh air and fuel prior to combustion.

- Nominal ratios of fresh air, fuel, and residual gas are constant, but precise values in each cycle can vary as independent random variables about nominal values (typically with a Gaussian distribution).

- Combustion efficiency (CE, fraction of fuel burned) depends solely on mean equivalence ratio prior to combustion.

- Combustion efficiency has four characteristic behaviors depending on the mean equivalence ratio $(\phi)$ :
(1) $\phi>1$, CE limited by available air; (2) $\phi_{\mathfrak{v}} \leq \phi \leq 1$, $\mathbf{C E}=1 ;$ (3) $\phi_{1}<\phi<\phi_{u}, \mathbf{C E}$ varies from 0 at $\phi_{1}$ to 1 at $\phi_{u}$ as a decaying function, typically linear or sigmoidal; (4) $\phi<\phi_{1}$ (true lean limit), $C E=0$.

Inter-cycle coupling occurs through the residual gas, which affects trailing cycles by altering the mean equivalence ratio prior to combustion. The steep drop in CE with $\phi$ is a key feature. Other processes such as injector noise, fuel vaporization, and turbulent mixing are treated as random parameter variations. Applying the above assumptions to cycle-by-cycle fuel and air material balances yields:

$$
\begin{aligned}
& m(i+1)=m(i)(1-C E(i)) F+(1-F) m_{f} \\
& a(i+1)=(a(i)-\mathbf{R} \cdot \mathbf{C E}(\mathbf{i}) \cdot \mathbf{m}(\mathbf{i})) \mathbf{F}+(1-\mathbf{F}) \mathbf{a}_{\mathrm{f}}
\end{aligned}
$$

where $\mathbf{a}(\mathbf{i})=$ mass of air before the ith burn; $\mathbf{a}_{\mathrm{f}}=$ maximum mass of fresh air injected with no residual gas; $m(i)=$ mass of fuel before the ith burn; $m_{f}=$ maximum mass of fresh fuel injected with no residual gas; $C E(i)=$ cycle i combustion efficiency; $\mathbf{F}$ = residual gas fraction; $\mathbf{R}$ = stoichiometric air/fuel mass ratio.

Masses $a_{f}$ and $m_{f}$ are related by as-injected equivalence ratio $\left(\phi_{\mathrm{f}}=\mathrm{m}_{\mathrm{f}} / \mathbf{a}_{\mathrm{f}}\right)$ using the constant mole condition and total cylinder volume. The parameter actually specified is $\phi_{f}$, from which $a_{f}$ and $m_{f}$ are determined. The parameter $\mathbf{R}$ is fixed by fuel composition. Residual gas fraction (F) is a function of cylinder design and operating condition (e.g., F is typically higher at idle, low-load). Combustion efficiency parameters $\phi_{u}$ and $\phi_{1}$ probably depend on the fuel and mixing details. We are currently researching appropriate parameter values, and in the future we expect to directly fit parameters with experimental data.

\footnotetext{
*Corresponding author, Proceedings of the 1996 Technical Meeting of the Central States Section of the Combustion Institute
} 
Reasonable parameter ranges are $0.1-0.3$ for $F, 0.6-0.55$ for $\phi_{a}$, and $0.55-0.5$ for $\phi_{1}$.

In the context of dynamical systems theory, Eqs. (1) and (2) are a two-dimensional mapping from one cycle to the next. Thus fuel and air masses in cycle $i+1$ prior to combustion are determined uniquely from fuel and air masses present before combustion in cycle $i$. This mapping is completely deterministic in the absence of parameter perturbations. Although (1) and (2) appear linear, the effect of $\mathbf{m}(\mathrm{i})$ on $\mathrm{CE}$ produces nonlinearity. We typically report model output as normalized mass of fuel consumed $\left(\Delta \mathbf{m} / \mathbf{m}_{f}\right)$, which is proportional to heat release.

One way to observe the effect of individual model parameters is to make plots such as Fig.1, which shows predicted fuel combustion as injected equivalence ratio is reduced from near stoichiometric to below $\phi_{1}$. This plot is produced by repeatedly iterating Eqs. (1) and (2) for fixed parameter values, beginning with an arbitrary initial condition. After initial transients die out, the results of several hundred iterates are plotted, and then the process repeated for different $\phi_{f}$. The final result is referred to as a bifurcation plot. See Moon[13] for detailed discussions. Although details change with parameter values, the same general trend is followed for a wide range of cases:

(1) near stoichiometric conditions, the amount of fuel burned in each cycle stabilizes to a single fixed value (a fixed point); (2) for $\phi_{f}$ below a critical value, the amount of fuel burned oscillates between two distinct values (a period 2 bifurcation); (3) for still lower $\phi_{f}$ combustion oscillations become more complex, leading to multi-period or chaotic patterns; (4) for $\phi_{f}$ below the lean limit, all combustion ceases. Adding noise to one or more parameters "blurs" the bifurcation sequence, but the same general pattern remains.

\section{Experimental}

We collected experimental data from a production V8 engine with standard port fuel injection connected to a DC motoring dynamometer. Injected fuel/air ratio was decreased from stoichiometric to very lean (where the engine was producing little torque). The nominal operating condition was $1200 \mathrm{RPM}, 20 \mathrm{ft}-\mathrm{lbs}, 20$ degrees BTC spark. We operated the dynamometer in speed control mode so as to keep the engine running at constant speed despite erratic combustion at very lean conditions. Feedback engine controllers were engaged to achieve an operating condition; once the condition was achieved the feedback controllers were shutoff and the engine run open-loop, except for dynamometer speed control. This assured to best experimental ability that combustion was minimally influenced by feedback controllers while the engine ran at constant speed. We recorded combustion pressure once/crank angle degree from a single cylinder and nominal operating conditions at a $50 \mathrm{~Hz}$ rate for over 2800 contiguous cycles. Cycle-by-cycle heat release was calculated by integrating the pressure data.

\section{Discussion}

For discussion purposes, we ran the model at near stoichiometric, moderately lean, and very lean fueling conditions for "typical" parameter values (e.g., $\mathbf{F}=0.25$, $\phi_{0}=0.60, \phi_{1}=0.53$, with linear decay in CE between $\phi_{u}$ and $\phi_{\nu}$. We also added Gaussian noise to $\phi_{\mathrm{f}}$ with a standard deviation of $2 \%$ about the nominal value. The above values were selected somewhat arbitrarily and were not intended to be exact "fits" for the experimental engine. Our intention is rather to compare predicted and observed trends.

Figures 2 and 3 compare model and experimental heatrelease frequency distributions for the three fueling conditions. Gaussian distributions with the same mean and standard deviation are also depicted for each case. For both model and experiment, near stoichiometric fueling is characterized by simple Gaussian fluctuations in heat release, reflecting the dominance of stochastic processes. Decreasing $\phi_{f}$ leads to deviations from Gaussian structure, reflecting an increasing influence from residual gas effects. In effect, the determinism amplifies and nonlinearly transforms the Gaussian perturbations.

Figures 4 and 5 illustrate similar model and experiment behavior using a "repeated measurement" statistic. This statistic quantifies the relative frequency that a given heat release observation will match a previous observation (within some tolerance) from $\mathbf{k}$ cycles in the past. If similar measurements separated by $\mathbf{k}$ cycles occur more frequently than measurements separated by one cycle, a "hidden" periodicity is implied. Figures 4 and 5 indicate that a period 2 pattern has emerged at moderately lean fueling. The "hidden" period 2 is revealed by elevated frequency peaks at multiple intervals of 2 . As in Fig. 1, the periodicity is "blurred", but the pattern is definitely present. No peaks are seen near stoichiometric fueling, where bifurcation has not occurred.

Figures 6 and 7 illustrate how the underlying periodicity interacts with noise to create classic symptoms of chaos. Both "return map sequences" depict how selected sets of engine cycle time sequences, successively map in relation to each other. (The reader is encouraged to consult [13] on the construction and meaning of such maps.) The coordinates $X(i)$ and $X(i+2)$ are heat release pairs for the ith and $i t h+2$ cycles, respectively. Note that experimental heat releases are in Joules, but model values are 
normalized as described earlier. The light shaded points represent the map for all of the observations. The darker points are from a selected set of engine cycles that are tracked sequentially in time in the successive frames (iterate 0 , iterate 1 and so on). Indices listed in the figure captions represent the beginning cycle numbers (for the frame marked iterate 0 ) of the dark points. The patterns followed by the dark points reveal defining characteristics of deterministic chaos. Specifically, we see clear evidence for period 2 fixed points and their associated stable and unstable manifolds. As shown, the darker points collapse together along the stable manifolds (linear point clusters with shallow slopes) onto the fixed points, oscillate between the fixed points for several iterates, and then diverge from the fixed points (and each other) along unstable manifolds (linear point clusters with steep slopes). The occurrence of these patterns in both the model and experiment implies similar dynamics. The chance for finding such patterns in random data is infinitesimally small.

\section{Conclusions}

The model captures essential trends in experimental observations. Chaotic data analysis confirms the presence of chaos with dynamical noise in the observed engine behavior consistent with the model results. The ability of our model to exhibit both stochastic and deterministic dominated regimes may help explain apparent discrepancies in previous observations.

\section{Acknowledgments}

This work is supported by the U.S. Department of Energy, Office of Energy Research and the Ford Motor Company.

\section{References}

1. Clerk, D., The Gas Engine, 1st ed., John Wiley and Sons (1893).

2. Belmont, M.R., Hancock, M.S., and Buckingham, D.J., SAE Paper No. 860324 (1986).

3. Brehob, D.D. and Newman, C.E., SAE Paper No. 922165 (1992).

4. Daily, J.W., Comb. Sci. and Tech., 57, pp 149-162 (1988).

5. Grunefeld, G., Beushausen, V., Andersen, P., and Hentschel, W., SAE Paper No. 941880 (1994).

6. Hamai, K., Kawajiri, H., Ishizuka, T., and Nakai, M., 21st Int. Symposium on Combustion, pp 505-512 (1986).

7. Hill, P.G. and Kapil, A., Comb. and Flame, 78, pp 237-247 (1989).

8. Kantor, J.C., Science, 224, pp 1233-1235 (1984).

9. Kumar, S., De-Zylva, M.D., and Watson, H.C., SAE Paper No. 912454 (1991).

10. Martin, J.K., Plee, S.L., and Remboski, D.J., Jr., SAE Paper No. 880201 (1988).

11. Moriyoshi, Y., Kanimoto, T., and Yagita, M., SAE Paper No. 930066 (1993).

12. Ozdor, N., Dulger, M., and Sher, E., SAE Paper No. 940987 (1994).

13. Moon, F.C., Chaotic and Fractal Dynamics, John Wiley and Sons (1992).

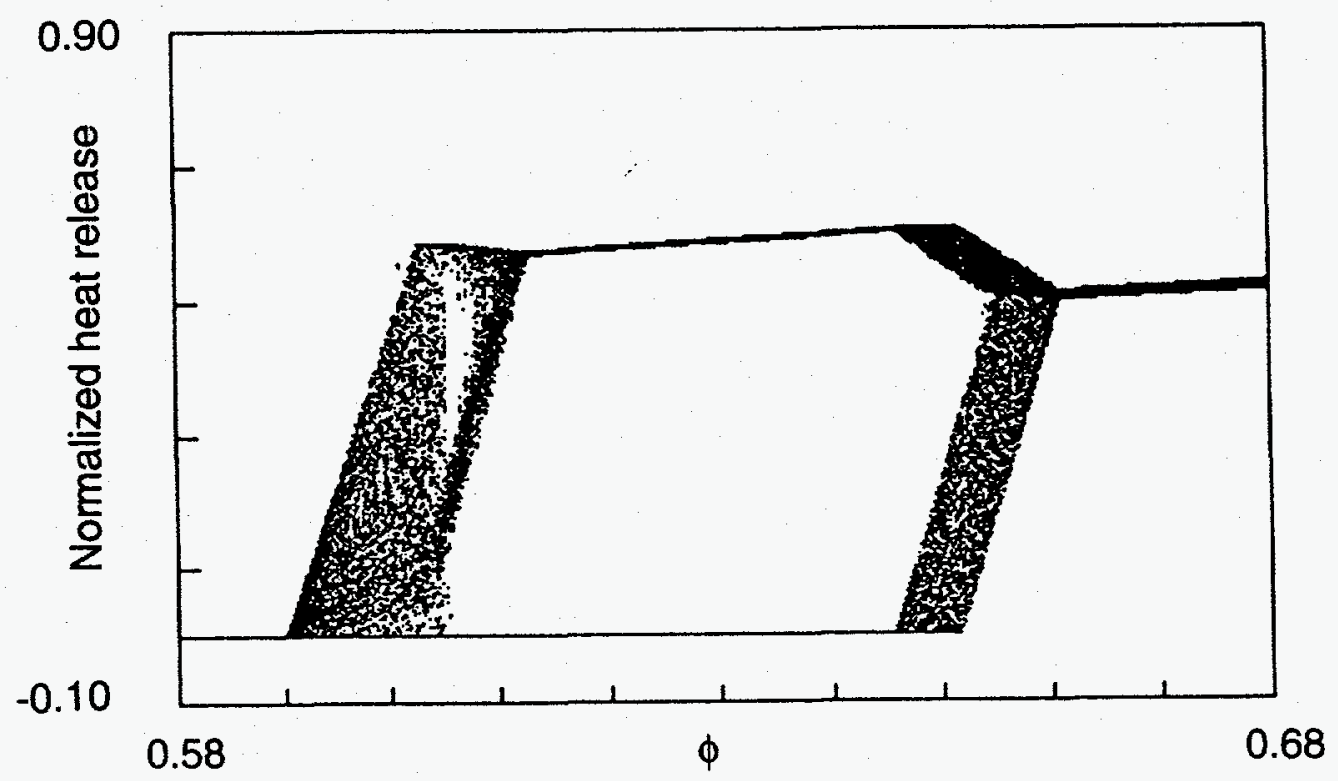

Figure 1. Equivalence ratio bifurcation plot for model with $F=0.21, R=14.6, \phi_{u}=0.60, \phi_{1}=0.59$. Random perturbations have been added to $\mathbf{F}$. 

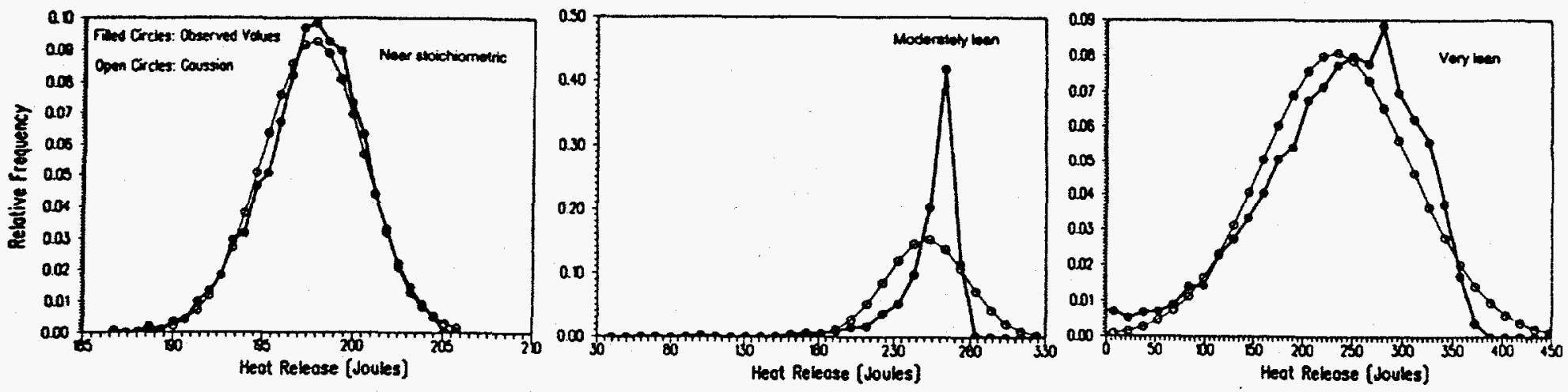

Figure 2. Experimental heat release frequency histograms for near stoichiometric, moderately lean. and very lean fueling. Note evolution away from Gaussian structure with decreasing equivalence ratio.
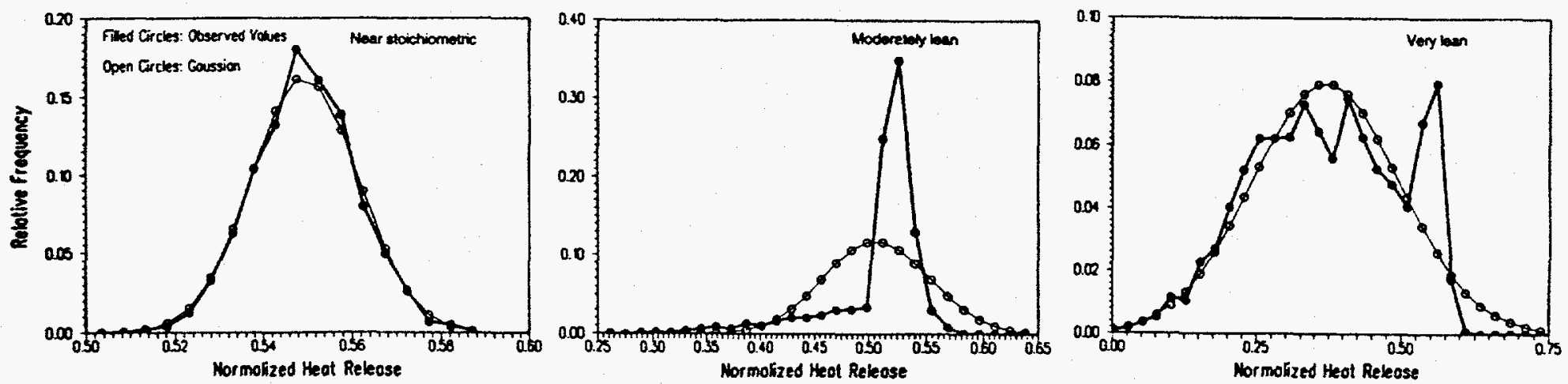

Figure 3. Model heat release frequency histograms for near stoichiometric, moderately lean, and very lean fueling. Dynamic noise is included by adding Gaussian noise to the as-injected fuel/air ratio. Note similarity to Fig. 2.

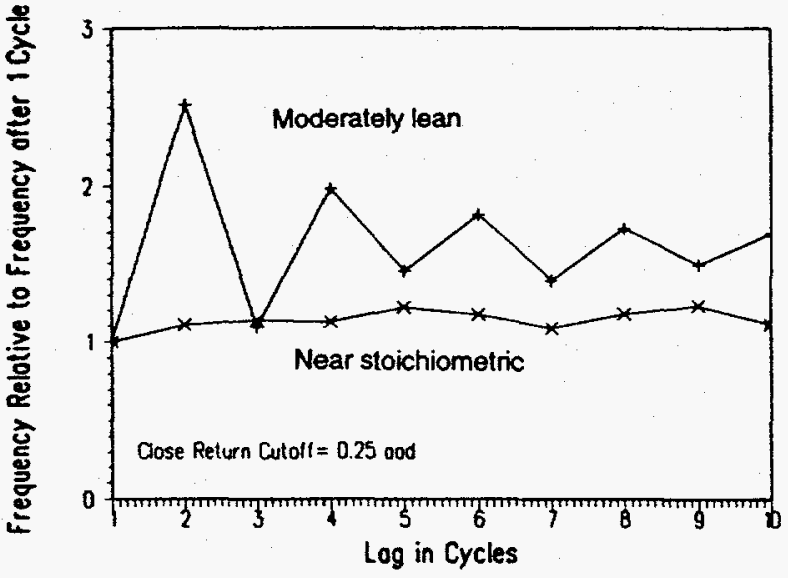

Figure 4. Experimental relative return frequency plot for moderately lean fueling. Peaks at multiples of 2-cycle lag indicate presence of noisy period 2.

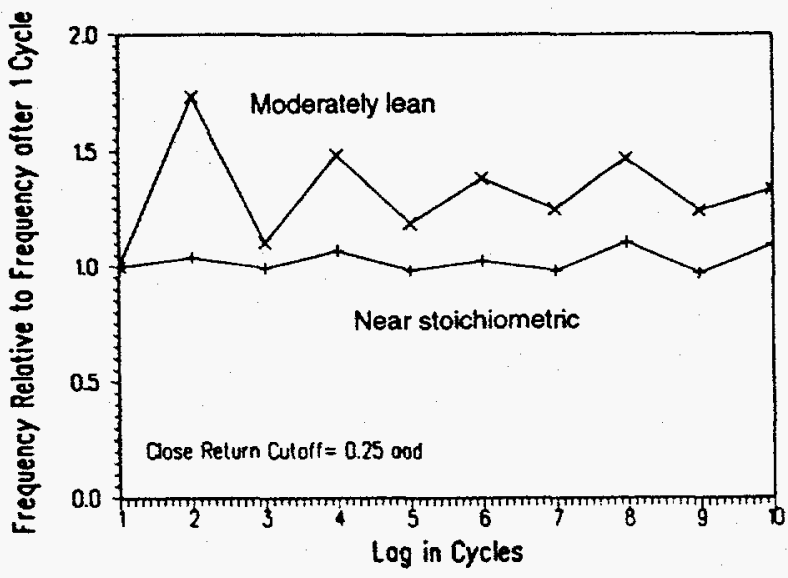

Figure 5. Model relative return frequency plot for moderately lean fueling. Note similarity to Fig. 4. 

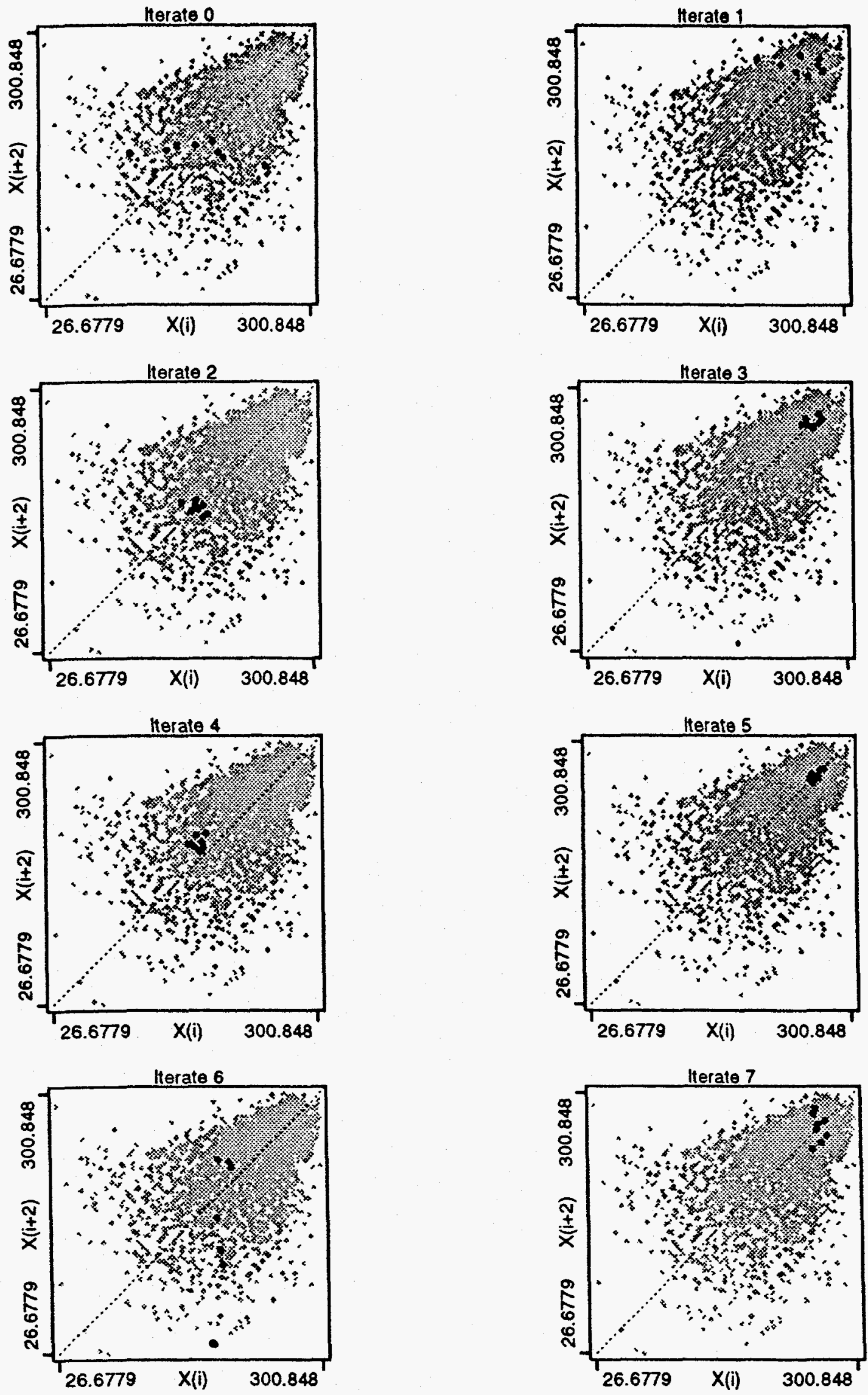

Figure 6. Experimental return map sequences for moderately lean fueling. Period 2 fixed points and their stable and unstable manifolds are clearly visible. Starting indices: 217, 284, 357, 448, 857, 1047, 1523, 2440. 

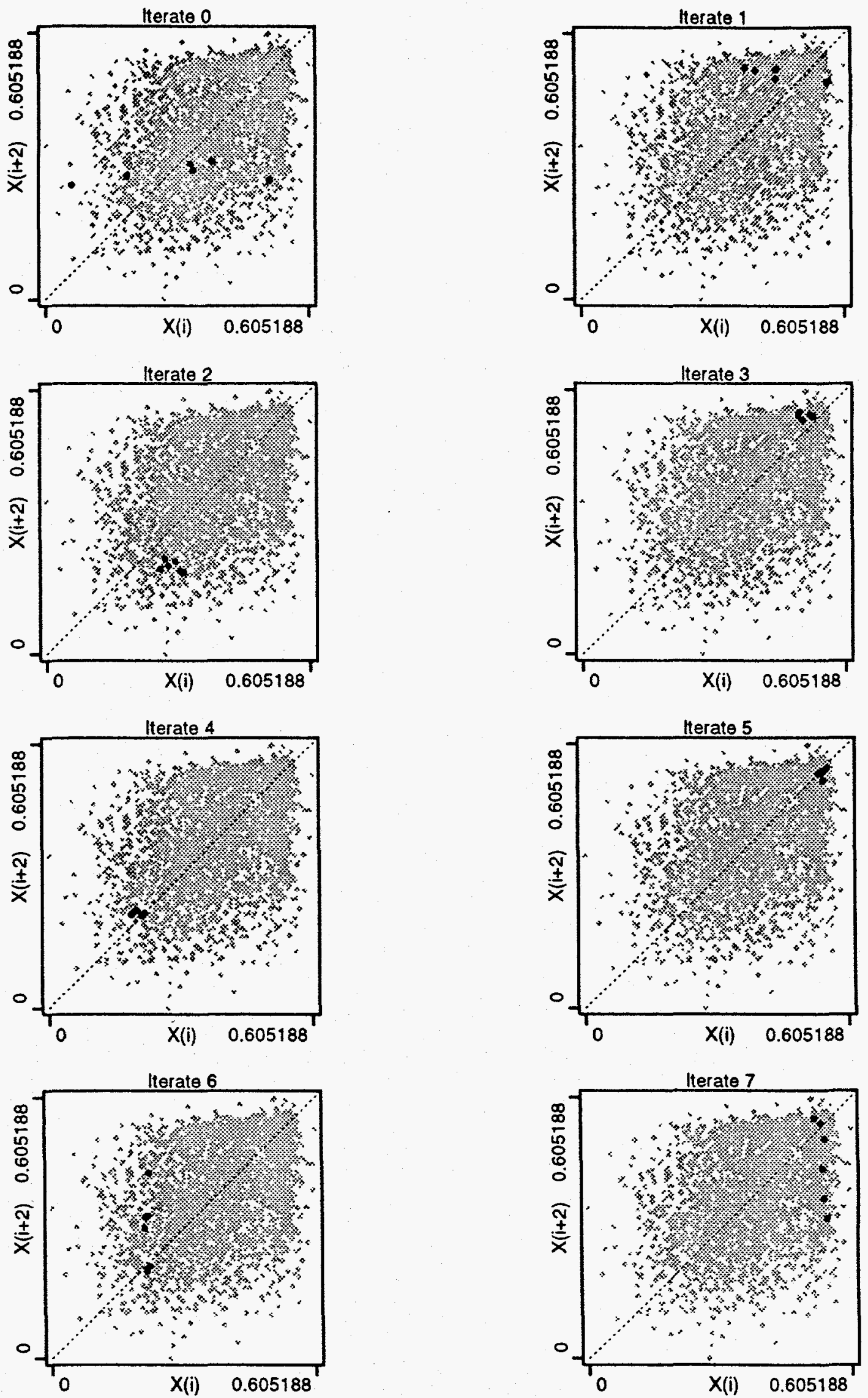

Figure 7. Model return map sequences for moderately lean fueling. Note similarity to the experimental patterns in Fig. 6. Starting indices: 670, 1031, 1086, 1626, 2163, 2292. 